\title{
Prognostic impacts of EGFR mutation status and subtype in patients with surgically resected lung adenocarcinoma
}

\author{
Kazuya Takamochi, MD, Shiaki Oh, MD, Takeshi Matsunaga, MD, and Kenji Suzuki, MD
}

\section{ABSTRACT}

Objective: Epidermal growth factor receptor (EGFR) gene mutation status is a well-established predictor of the efficacy of EGFR tyrosine-kinase inhibitor (TKI) therapy in patients with non-small cell lung cancer. Recently, differences in EGFR mutation subtypes have been reported to be associated with the efficacy of EGFR-TKI therapy. The prognostic impact of EGFR mutation status and subtypes remains controversial, however.

Methods: We retrospectively reviewed 939 patients with surgically resected adenocarcinomas who underwent EGFR mutation status analysis between January 2010 and December 2014. Overall survival (OS) and recurrence-free survival (RFS) were compared according to pathological stage, EGFR mutation status, and EGFR mutation subtype using the log-rank test. Independent prognostic factors for OS and RFS were identified by multivariate analysis using the Cox proportional hazards model.

Results: The median duration of follow-up was 48 months. We found that positive EGFR mutation status was significantly associated with longer OS and RFS in all patients and was associated with longer OS in patients in pathological stage I; however, there were no significant differences in OS and RFS between patients with exon $21 \mathrm{~L} 858 \mathrm{R}$ mutations and those with exon 19 deletions. In a Cox regression model for OS, EGFR mutation status was a significant prognostic factor that was independent of well-established prognostic factors, including age, pathological stage, vascular invasion, lymphatic permeation, and serum carcinoembryonic antigen level.

Conclusions: Positive EGFR mutation status is a favorable prognostic factor in patients with surgically resected lung adenocarcinomas; however, EGFR mutation subtype (exon 21 L858R mutation or exon 19 deletion) exhibits no prognostic impact. (J Thorac Cardiovasc Surg 2017;154:1768-74)

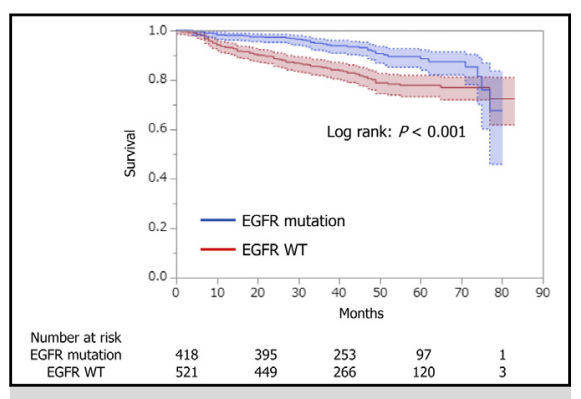

Improved survival associated with EGFR mutant lung tumors relative to wild-type tumors.

\section{Central Message}

Positive EGFR mutation status is a favorable prognostic factor in patients with surgically resected lung adenocarcinomas; however, EGFR mutation subtype has no prognostic impact.

\section{Perspective}

We have shown that positive EGFR mutation status is a favorable prognostic factor independent of TNM stage in patients with surgically resected lung adenocarcinomas. During the next revision of the TNM classification of lung cancer, cancer genotype might be adapted as a new category, and in future clinical trials for adjuvant therapy, stratification by EGFR mutation status might be necessary to avoid bias.

See Editorial Commentary page 1775.

See Editorial page 1766.
Mutations in the epidermal growth factor receptor gene $(E G F R)$ represent one of the most frequent driver-

From the Department of General Thoracic Surgery, Juntendo University School of Medicine, Tokyo, Japan.

This work was supported in part by a Grant-in-Aid for Cancer Research from the Ministry of Health, Labor, and Welfare of Japan and the Smoking Research Foundation.

Received for publication Jan 26, 2017; revisions received June 7, 2017; accepted for publication June 30, 2017; available ahead of print Aug 18, 2017.

Address for reprints: Kazuya Takamochi, MD, Department of General Thoracic Surgery, Juntendo University School of Medicine, 1-3, Hongo 3-chome, Bunkyo-ku, Tokyo 113-8431, Japan (E-mail: ktakamo@juntendo.ac.jp). $0022-5223 / \$ 36.00$

Copyright (c) 2017 by The American Association for Thoracic Surgery http://dx.doi.org/10.1016/j.jtcvs.2017.06.062 oncogene alterations in non-small cell lung cancer (NSCLC). EGFR mutations are more frequently found in females, those who have never smoked, patients with adenocarcinoma, and individuals of East Asian descent. The frequency of EGFR mutations in nonadenocarcinoma NSCLCs is quite low $(<2 \%) .{ }^{1}$ The EGFR tyrosine kinase inhibitors (TKIs) gefitinib and erlotinib, which specifically

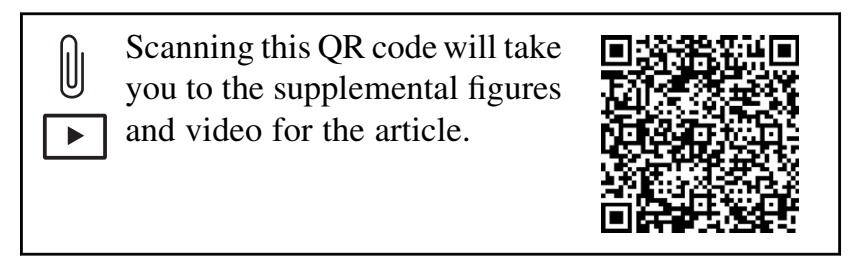



Abbreviations and Acronyms
CEA = carcinoembryonic antigen
CI = confidence interval
CT = computed tomography
EGFR = epidermal growth factor receptor
MRI = magnetic resonance imaging
NSCLC $=$ non-small cell lung cancer
OS = overall survival
PET $={ }^{18}$ F-fluoro-2-deoxy-glucose positron emission tomography
RFS = recurrence-free survival
TKI = tyrosine kinase inhibitor
WT = wild type

block the EGFR-dependent pathway, were initially approved as molecular-targeting drugs for their use in the treatment of NSCLC. ${ }^{2}$ EGFR-mutated NSCLC has become a clinically relevant molecular subset, because patients with NSCLC harboring activating EGFR mutations (exon 21 L858R mutation or exon 19 deletions) benefit from EGFR-TKI treatment, unlike those with EGFR wild-type (WT) tumors. ${ }^{3,4}$

Both the presence of EGFR mutations and differences in EGFR mutation subtypes (exon 21 L858R mutation or exon 19 deletions) have been associated with the efficacy of EGFR-TKIs. A meta-analysis of 8 clinical trials and 5 retrospective studies using EGFR-TKIs for advanced EGFR-mutated NSCLCs identified exon 19 deletion as associated with longer progression-free survival compared with exon 21 L858R mutation. ${ }^{5}$ Therefore, both EGFR mutation status and $E G F R$ mutation subtype are considered significant predictors of the efficacy of EGFR-TKI therapy in NSCLC.

In contrast, the prognostic impact of both EGFR mutation status and EGFR subtype in patients with NSCLC remains controversial. A systematic review with a meta-analysis based on data from 16 studies (n = 3337) showed that EGFR mutations were not a prognostic factor in patients with surgically resected NSCLC $^{6}$; however, all of the studies included in the meta-analysis were of a retrospective design, included only small numbers of patients, had a relatively short follow-up period, and involved heterogeneous treatments for recurrent disease (especially regarding the use of EGFR TKI). Therefore, as a matter of course, further study is needed to confirm the results. The prognostic impact of EGFR mutation subtypes (exon 21 L858R point mutation or exon 19 deletions) in patients with NSCLC has not been well established, given the paucity of studies investigating the prognostic impact of EGFR mutation subtypes in patients with early-stage NSCLC who underwent surgical resection. ${ }^{7-9}$ In the present study, we explored the prognostic impacts of EGFR mutation status and EGFR subtype in patients with surgically resected lung adenocarcinomas.

\section{METHODS \\ Patients}

Between January 2010 and December 2014, a total of 1018 patients with lung adenocarcinomas underwent complete surgical resection at Juntendo University School of Medicine (Tokyo, Japan). Among these patients, we retrospectively reviewed clinicopathological data for 939 consecutive patients with lung adenocarcinomas who had available data on $E G F R$ mutation status. A flowchart illustrating the selection of the study cohorts is shown in Figure 1.

Preoperative staging was routinely performed with computed tomography (CT) scans of the chest and upper abdomen and ${ }^{18} \mathrm{~F}$-fluoro2-deoxy-glucose positron emission tomography (PET)/CT scans of the chest and upper abdomen. Cranial CT scanning or magnetic resonance imaging (MRI) was performed based on clinical signs and symptoms. The patients were staged based on the seventh edition of the International Association for the Study of Lung Cancer TNM classification for lung cancer. ${ }^{10}$ No patient receive induction chemotherapy or radiotherapy, or treatments with EGFR-TKI as induction or adjuvant therapy.

\section{Follow-up and Diagnosis of Recurrence}

Follow-up examinations were performed every 3 or 4 months for the first 3 years and then every 6 months thereafter. Chest X-rays and biochemical examination of blood, including for carcinoembryonic antigen (CEA), were performed at each visit to the outpatient department. Chest CT or PET/CT scans were performed annually. Cranial CT scans and MRI were performed as necessary. Recurrence was diagnosed based on radiologic evidence of cancer relapse on surveillance imaging and/or pathological evidence according to tumor biopsy analysis.

\section{EGFR Mutation Analysis}

We routinely analyzed $E G F R$ mutations for lung cancer patients who underwent surgery for a research purpose. Genomic DNA was extracted from cubes $\left(3\right.$ to $5 \mathrm{~mm}^{3}$ ) of frozen, fresh lung cancer tissue samples from surgically resected specimens. The peptide nucleic acid-locked nucleic acid polymerase chain reaction clamp method ${ }^{11}$ was used to identify EGFR mutations G719A, G719C, and G719A in exon 18; all deletion genotypes in exon 19; T790M in exon 20; and L858R and L861Q in exon 21.

Approval for collection of patient specimens from the tissue bank in our department was obtained from the Institutional Review Board of Juntendo University School of Medicine (no. 2014172). Individual patient consent was obtained for the procurement of tissue for research purposes before surgery. This retrospective study was performed under a waiver of authorization approved by the Institutional Review Board.

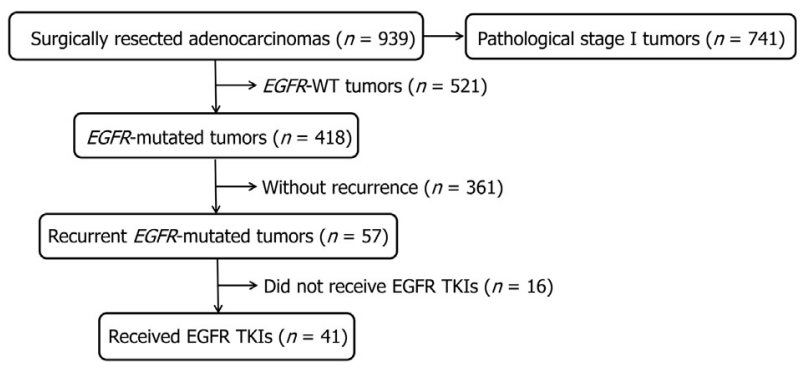

FIGURE 1. Flowchart show the selection of the study cohort. EGFR, Epidermal growth factor receptor; WT, wild type; TKI, tyrosine-kinase inhibitor. 


\section{Statistical Analysis}

Overall survival (OS) and recurrence-free survival (RFS) were analyzed by the Kaplan-Meier method, and curve differences were tested using the log-rank test according to pathological stage, EGFR mutation status, and EGFR mutation subtypes. The duration of OS was defined as the interval between the date of surgical intervention and the date of either death or the last follow-up. The duration of RFS was defined as the interval between the date of surgical intervention and the date of recurrence, death from any cause, or the last follow-up. Univariate and multivariate analyses of independent prognostic factors in each cohort were performed using the Cox proportional hazards model. All significant variables $(P<.05)$ in the univariate analyses were entered into the multivariate analyses using a stepwise variable selection procedure to adjust for potential confounding factors.

A $P$ value $<.05$ was considered to indicate statistical significance. All statistical analyses were performed using SPSS version 20.0 (IBM, Armonk, NY).

\section{RESULTS}

EGFR mutations were detected in 418 of the 939 patients with lung adenocarcinoma ( $45 \%)$, with the point mutation L858R in exon 21 detected in 222 of $418(53 \%)$ $E G F R$-mutated tumors and exon 19 deletions detected in 161 of these tumors (39\%). Other minor EGFR mutations were detected in 23 tumors $(6 \%)$, including mutations of exon 18 G719A in 8 , exon 18 G719S in 5, exon 18 G719C in 3, exon 21 L861Q in 7, and exon 20 T790M in 4. Double mutations were found in 12 tumors $(3 \%)$, including exon $21 \mathrm{~L} 858 \mathrm{R}$ and exon $20 \mathrm{~T} 790 \mathrm{M}$ in 8 and exon $20 \mathrm{~T} 790 \mathrm{M}$ and exon 19 deletion, exon $20 \mathrm{~T} 790 \mathrm{M}$ and exon 18 G719A, exon 18 G719A and exon 21 L861Q, and exon $18 \mathrm{G} 719 \mathrm{~S}$ and exon 21 L861Q in 1 each. Interestingly, exon $20 \mathrm{~T} 790 \mathrm{M}$, which confers resistance to EGFR-TKI, ${ }^{12}$ was consistently coupled with other mutations. Patient characteristics are summarized in Table 1. Female sex $(P<.001)$, nonsmoker $(P<.001)$, pathological stage I tumor $(P=.001)$, and tumor without vascular invasion $(P=.002)$ were more frequent in patients with $E G F R$-mutated tumors compared with patients with $E G F R$ WT tumors.

To evaluate the prognostic impact of EGFR mutation status, we analyzed OS and RFS in 939 patients, with a median follow-up of 48 months. The 3-/5-year OS rates were $95 \% \quad[95 \%$ confidence interval (CI), $92 \%-97 \%] / 89 \%$ (95\% CI, 84\%-92\%) in the 418 patients with EGFR-mutated tumors and $85 \% \quad(95 \% \quad \mathrm{CI}$, $82 \%-88 \%) / 78 \%(95 \%$ CI, $73 \%-82 \%)$ in the 521 patients with EGFR WT tumors. Positive EGFR mutation status was significantly associated with longer OS in all patients $(P<.001$; Figure 2, A). The 3-/5-year RFS rates were $85 \%$ (95\% CI, $81 \%-88 \%) / 81 \%$ (95\% CI, $76 \%-85 \%)$ in the 418 patients with EGFR-mutated tumors and $78 \%$ $(95 \%$ CI, $75 \%-82 \%) / 72 \%(95 \%$ CI, 68\%-77\%) in the 521 patients with EGFR WT tumors, with positive EGFR mutation status significantly associated with longer RFS in all patients $(P=.002$; Figure $2, B)$.
TABLE 1. Patient characteristics

\begin{tabular}{|c|c|c|c|}
\hline Characteristic & $\begin{array}{c}\text { EGFR } \\
\text { mutation, } \\
\text { n }(\%) \\
\end{array}$ & $\begin{array}{c}\text { EGFR } \\
\text { WT, } \\
\text { n }(\%)\end{array}$ & $\begin{array}{c}P \\
\text { value }\end{array}$ \\
\hline Total number & 418 & 521 & \\
\hline Age, y, median (range) & $67(33-78)$ & $67(22-88)$ & .266 \\
\hline $\begin{array}{l}\text { Sex, n }(\%) \\
\quad \text { Male } \\
\text { Female }\end{array}$ & $\begin{array}{l}158(38) \\
260(62)\end{array}$ & $\begin{array}{l}299(57) \\
222(43)\end{array}$ & $<.001$ \\
\hline $\begin{array}{l}\text { Smoking status, n (\%) } \\
\text { Nonsmoker } \\
\text { Smoker } \\
\text { Unknown }\end{array}$ & $\begin{array}{c}281(67) \\
134(32) \\
3(1)\end{array}$ & $\begin{array}{c}212(53) \\
304(46) \\
5(1)\end{array}$ & $<.001$ \\
\hline $\begin{array}{l}\text { Serum CEA level, n (\%) } \\
\text { Normal } \\
\text { Elevated } \\
\text { Unknown }\end{array}$ & $\begin{array}{c}233(56) \\
179(43) \\
6(1)\end{array}$ & $\begin{array}{c}265(51) \\
250(48) \\
6(1)\end{array}$ & .122 \\
\hline $\begin{array}{l}\text { Pathological stage, n (\%) } \\
\text { I } \\
\text { II-IV }\end{array}$ & $\begin{array}{r}350(84) \\
68(16)\end{array}$ & $\begin{array}{l}391(75) \\
130(25)\end{array}$ & .001 \\
\hline $\begin{array}{l}\text { Lymphatic permeation, n (\%) } \\
\text { Present } \\
\text { Absent }\end{array}$ & $\begin{array}{r}69(17) \\
349(83)\end{array}$ & $\begin{array}{r}97(19) \\
424(81)\end{array}$ & .399 \\
\hline $\begin{array}{l}\text { Vascular invasion, } \mathrm{n}(\%) \\
\text { Present } \\
\text { Absent }\end{array}$ & $\begin{array}{r}67(16) \\
351(84)\end{array}$ & $\begin{array}{l}127(24) \\
394(76)\end{array}$ & .002 \\
\hline
\end{tabular}

$\overline{E G F R, \text { Epidermal growth factor receptor; } W T \text {, wild type; } C E A \text {, carcinoembryonic }}$ antigen.

The 3-/5-year OS rates were $98 \%$ (95\% CI, 96\%-99\%)/ $94 \%(95 \%$ CI, 90\%-97\%) in the 350 patients with EGFR-mutated pathological stage I tumors and 93\% (95\% CI, $90 \%-95 \%) / 87 \%$ (95\% CI, 83\%-91\%) in the 391 patients with EGFR WT pathological stage I tumors. Positive $E G F R$ mutation status was significantly associated with longer OS in patients in pathological stage I $(P=.003$; Figure E1, $A$ ). In addition, the 3-/5-year RFS rates were 93\% (95\% CI, 90\%-96\%)/89\% (95\% CI, 84\%-93\%) in the 350 patients with EGFR-mutated pathological stage I tumors and $90 \%(95 \% \mathrm{CI}, 86 \%-93 \%) / 84 \%(95 \% \mathrm{CI}$, $80 \%-88 \%$ ) in the 391 patients with EGFR WT pathological stage I tumors. Here again, positive EGFR mutation status was associated with longer RFS in the patients with pathological stage I tumors; however, the difference was not statistically significant $(P=.065$; Figure E1, $B)$. Cox regression analysis of prognostic factors for OS in all patients identified EGFR mutation status as a significant prognostic factor independent of well-established prognostic factors including age, serum CEA level, pathological stage, vascular invasion, and lymphatic permeation (Tables 2 and 3). Cox regression analysis of prognostic factors for RFS in all patients identified EGFR mutation status as a significant prognostic factor in the univariate analysis, but not in the multivariate analysis (Tables 2 and 3). 
OS

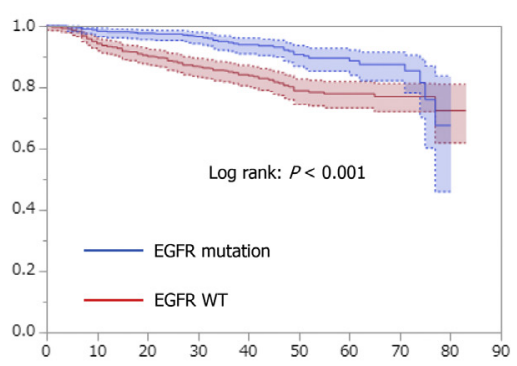

Number at risk

A EGFR mutation

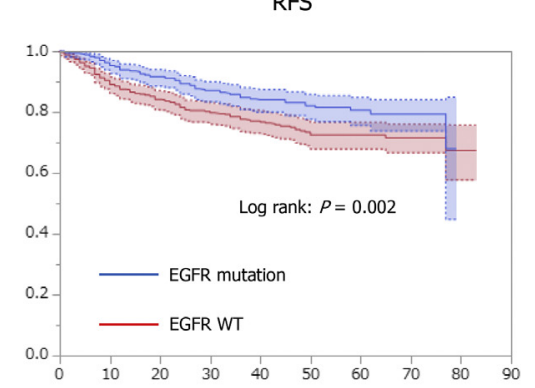

$\begin{array}{lllll}418 & 370 & 222 & 85 & 0 \\ 521 & 422 & 245 & 111 & 3\end{array}$

FIGURE 2. Overall survival (A) and recurrence-free survival (B) in all patients according to EGFR mutation status. $O S$, Overall survival; $R F S$, recurrencefree survival; $E G F R$, epidermal growth factor receptor; $W T$, wild type.

To evaluate the prognostic impact of EGFR mutation subtypes, we analyzed OS and RFS rates for patients with $E G F R$-mutated tumors in 3 groups stratified by EGFR mutation subtypes: exon 19 deletions, L858R, and other subtypes, including minor mutations or double mutations. In 418 patients with EGFR-mutated tumors, we found no significant differences in OS and RFS rates according to mutation subtype (Figure 3, A: exon 19 deletions vs L858R, $P=.172$; exon 19 deletions vs other subtypes, $P=.056$; L858R vs other subtypes, $P=.300$; Figure 3, $B$ : exon 19 deletions vs L858R, $P=.646$; exon 19 deletions vs other subtypes, $P=.961 ;$ L858R vs other subtypes, $P=.696)$.

Postoperative recurrences developed in 57 patients with EGFR-mutated tumors, including 10 locoregional only, 31 distant only, and 16 locoregional and distant. Among these 57 patients, 41 were treated with EGFR-TKIs following recurrence, and OS time tended to be longer in these patients compared with that in 16 patients not treated with EGFR-TKIs; however, the difference was not statistically significant $(P=.173$; Figure 4$)$. In the 41 patients who received EGFR-TKI treatment, both OS and survival

TABLE 2. Univariate analyses (Cox proportional hazards model) of prognostic factors for OS and RFS in all 939 patients

\begin{tabular}{|c|c|c|c|c|c|c|c|}
\hline \multirow[b]{2}{*}{ Factor } & \multirow[b]{2}{*}{ Value } & \multicolumn{3}{|c|}{ OS } & \multicolumn{3}{|c|}{ RFS } \\
\hline & & HR & $95 \% \mathrm{CI}$ & $P$ value & HR & $95 \%$ CI & $P$ value \\
\hline Age, y, median (range) & $67(22-88)$ & 1.05 & $1.03-1.07$ & $<.001$ & 1.03 & $1.01-1.04$ & $<.001$ \\
\hline Sex, n $(\%)$ & & & & .001 & & & $<.001$ \\
\hline Male & 457 (49) & 1.83 & $1.29-2.60$ & & 1.83 & $1.37-2.44$ & \\
\hline Female & $482(51)$ & 1 & & & 1 & & \\
\hline Smoking status, $\mathrm{n}(\%)$ & & & & $<.001$ & & & $<.001$ \\
\hline Non-smoker & $493(53)$ & 1 & & & 1 & & \\
\hline Smoker & $438(46)$ & 2.18 & $1.52-3.11$ & & 1.87 & $1.40-2.49$ & \\
\hline Serum CEA level, n (\%) & & & & $<.001$ & & & $<.001$ \\
\hline Normal & $498(53)$ & 1 & & & 1 & & \\
\hline Elevated & $429(46)$ & 3.45 & $2.34-5.08$ & & 2.96 & $2.18-4.01$ & \\
\hline Pathological stage, n (\%) & & & & $<.001$ & & & $<.001$ \\
\hline I & $741(79)$ & 1 & & & 1 & & \\
\hline II-IV & $198(21)$ & 6.72 & $4.73-9.54$ & & 8.26 & $6.20-11.0$ & \\
\hline Lymphatic permeation, $\mathrm{n}(\%)$ & & & & $<.001$ & & & $<.001$ \\
\hline Present & $166(18)$ & 4.61 & $3.27-6.51$ & & 4.97 & $3.75-6.59$ & \\
\hline Absent & $773(82)$ & 1 & & & 1 & & \\
\hline Vascular invasion, $\mathrm{n}(\%)$ & & & & $<.001$ & & & $<.001$ \\
\hline Present & $194(21)$ & 4.45 & $3.16-6.27$ & & 5.53 & $4.17-7.32$ & \\
\hline Absent & $745(79)$ & 1 & & & 1 & & \\
\hline EGFR mutation, $\mathrm{n}(\%)$ & & & & $<.001$ & & & .003 \\
\hline Positive & $418(45)$ & 0.45 & $0.31-0.66$ & & 0.64 & $0.48-0.85$ & \\
\hline Negative & $521(55)$ & 1 & & & 1 & & \\
\hline
\end{tabular}

$O S$, Overall survival; $R F S$, recurrence-free survival; $H R$, hazard ratio; $C I$, confidence interval; $C E A$, carcinoembryonic antigen; $E G F R$, epidermal growth factor receptor. 
TABLE 3. Multivariate analyses (Cox proportional hazards model) of prognostic factors for OS and RFS in all 939 patients

\begin{tabular}{|c|c|c|c|c|c|c|c|}
\hline \multirow[b]{2}{*}{ Factor } & \multirow[b]{2}{*}{ Value } & \multicolumn{3}{|c|}{ OS } & \multicolumn{3}{|c|}{ RFS } \\
\hline & & HR & $95 \%$ CI & $P$ value & HR & $95 \%$ CI & $P$ value \\
\hline Age, y, median (range) & $67(22-88)$ & 1.03 & $1.01-1.05$ & .001 & 1.02 & $1.00-1.03$ & .041 \\
\hline Serum CEA level, n (\%) & & & & .006 & & & .008 \\
\hline Normal & $498(53)$ & 1 & & & 1 & & \\
\hline Elevated & $429(46)$ & 1.78 & $1.18-2.69$ & & 1.56 & $1.12-2.16$ & \\
\hline Pathological stage, n (\%) & & & & $<.001$ & & & $<.001$ \\
\hline I & 741 (79) & 1 & & & 1 & & \\
\hline II-IV & $198(21)$ & 3.68 & $2.38-5.67$ & & 4.31 & $3.02-6.16$ & \\
\hline Lymphatic permeation, $\mathrm{n}(\%)$ & & & & .002 & & & .002 \\
\hline Present & $166(18)$ & 1.94 & $1.28-2.96$ & & 1.49 & $1.06-2.09$ & \\
\hline Absent & $773(82)$ & 1 & & & 1 & & \\
\hline Vascular invasion, $\mathrm{n}(\%)$ & & & & .006 & & & $<.001$ \\
\hline Present & $194(21)$ & 1.8 & $1.19-2.73$ & & 2.09 & $1.48-2.95$ & \\
\hline Absent & 745 (79) & 1 & & & 1 & & \\
\hline EGFR mutation, $\mathrm{n}(\%)$ & & & & .003 & & & - \\
\hline Positive & $418(45)$ & 0.56 & $0.38-0.82$ & & - & - & \\
\hline Negative & $521(55)$ & 1 & & & - & & \\
\hline
\end{tabular}

$O S$, Overall survival; $R F S$, recurrence-free survival; $H R$, hazard ratio; $C I$, confidence interval; $C E A$, carcinoembryonic antigen; $E G F R$, epidermal growth factor receptor.

periods following recurrence were longer in 19 patients with tumors harboring exon 19 deletion mutations compared with those in 18 patients with tumors harboring the L858R mutation; however, the differences were not statistically significant $(P=.256$ and .198 , respectively; Figure E2).

\section{DISCUSSION}

EGFR mutation status and subtype indisputably constitute significant predictive factors for the efficacy of EGFR-TKIs in advanced NSCLC; however, their prognostic role remains unclear, given the inconsistent results from previous studies of small and heterogeneous populations. ${ }^{6-8}$ Elucidation of the intrinsic prognostic impact of $E G F R$ mutation status and $E G F R$ mutation subtypes in patients with advanced EGFR-mutated NSCLC is currently considered impossible, because EGFR-TKIs are used as standard first-line treatment.
Furthermore, third-generation EGFR-TKIs were recently shown to prolong survival, even after the development of resistance to first- or second-generation EGFR-TKIs. ${ }^{13}$ Increasingly prolonged survival is expected for patients with advanced EGFR-mutated NSCLC. Even in the presence or absence of intrinsic prognostic impacts associated with EGFR mutation status and subtypes, their affect would be obscured by a definitive association with the efficacy of EGFR-TKIs. Therefore, the intrinsic prognostic roles of EGFR mutation status and subtype require investigation in patients with surgically resected early-stage NSCLC who did not receive perioperative therapy with EGFR-TKIs.

To the best of our knowledge, the present study is the largest-scale evaluation of patients with surgically resected $E G F R$-mutated tumors for evaluating the prognostic impact of EGFR mutation status and subtypes by analyzing both
OS

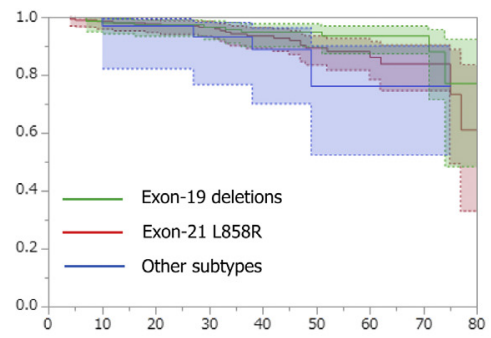

RFS

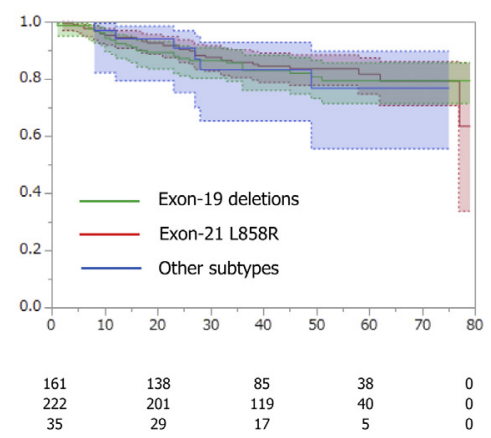

FIGURE 3. Overall survival (A) and recurrence-free survival (B) in patients with $E G F R$ mutated tumors according to EGFR mutation subtype (exon 19 deletions, exon 21 L858R mutation, or other mutations). OS, Overall survival; RFS, recurrence-free survival. 


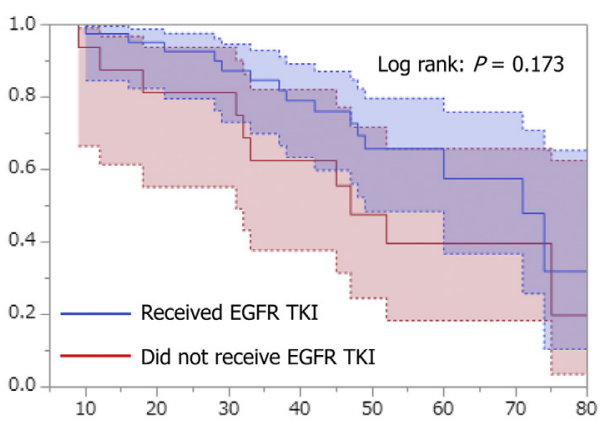

Number at risk

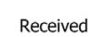

39

13
8

FIGURE 4. Comparison of overall survival in patients with EGFR mutated tumors who received EGFR-TKI treatment following recurrence and those who did not. EGFR, Epidermal growth factor receptor; $T K I$, tyrosine-kinase inhibitor.

OS and RFS rates reported to date. We found that positive EGFR mutation status was significantly associated with longer OS regardless of pathological stage in Kaplan-Meier analyses. Furthermore, multivariate analysis identified EGFR mutation status as a significant prognostic factor for OS independent of pathological stage. In addition, although the number of patients who received EGFR-TKIs for postoperative recurrence was small, there was no statistical difference in OS between whether patients with recurrent EGFR-mutated tumors received or did not receive EGFR-TKI treatment. The duration of OS might not be significantly influenced by EGFR-TKI treatment for postoperative recurrence; thus, we conclude that positive EGFR mutation status is a favorable prognostic factor in patients with surgically resected lung adenocarcinomas.

In contrast, we observed no significant differences in OS and RFS between patients with an exon 21 L858R mutation $(\mathrm{n}=222)$ and those with exon 19 deletions in EGFR-mutated tumors $(\mathrm{n}=161)$. In 41 patients who received EGFR-TKI treatment, both OS and survival following recurrence were longer in patients with tumors harboring exon 19 deletions ( $\mathrm{n}=19)$ compared with those with tumors harboring an L858R mutation $(\mathrm{n}=18$ ), although the differences were not statistically significant. Thus, we conclude that EGFR mutation subtype (exon 21 L858R mutation or exon 19 deletions) has no prognostic impact on patients with surgically resected lung adenocarcinomas. Larger-scale studies are needed to confirm these observations, however.

Although the underlying mechanism associated with the favorable prognostic impact of EGFR mutations in lung adenocarcinoma remains unclear, EGFR-mutated lung adenocarcinomas may be less likely to be affected by complex oncogenic processes, given that EGFR mutations provide strong growth advantages due to their constitutive activation. Therefore, EGFR-mutated lung adenocarcinoma cells need not acquire additional oncogenic mutations to enhance their biological malignancy via cell-based

The prognostic impact of EGFR mutation status and mutation subtypes in patients with surgically resected lung adenocarcinomas
Department of General Thoracic Surgery, Juntendo University School of Medicine

VIDEO 1. The importance and relevance of the present study. Video available at: http://www.jtcvsonline.org/article/S0022-5223(17)31574-X/fulltext. characteristics, such as invasive, antiapoptotic, and metastatic potentials. As a result, EGFR-mutated lung adenocarcinomas (non-smoking-related lung cancer) may acquire a lower degree of biological malignancy relative to that observed in smoking-related lung cancers exhibiting a high nonsynonymous mutation burden. ${ }^{14}$

TNM classifications for lung cancer were recently revised by the International Association for the Study of Lung Cancer's Staging Committee. ${ }^{15}$ Although the revised TNM staging system adapted categories based on prognostic factors associated with the anatomic extent of tumors, no consideration was given to the biological features of tumors, such as driver mutations. Recent advances in the understanding of NSCLC-related molecular biology likely will offer the potential for therapeutic success by matching systemic target therapies to cancer genotype. EGFR mutations offer not only predictive significance for EGFR-TKI treatment, but also improved prognostic significance from a biological standpoint for lung adenocarcinomas. Other alterations in driver oncogenes with a prognostic impact similar to that of EGFR mutations might be identified in the future. Therefore, regardless of how precise the classification system becomes, a TNM staging system based solely on the anatomic extent of tumors will become outdated and virtually irrelevant to clinical practice in the era of molecular-target therapy. In the next revision to the TNM classification of lung cancer, cancer genotype might be adapted as a new category.

Several clinical trials have reported higher chemotherapy response rates in the presence of EGFR mutations in both first-line and second-line advanced NSCLC settings. ${ }^{16,17}$ In phase III studies of adjuvant gefitinib (BR19) and erlotinib (RADIANT) for patients with completely resected stage IB to IIIA NSCLCs, EGFR-TKI therapy failed to show a survival benefit compared with placebo in subgroups of patients with EGFR-mutated tumors. ${ }^{18,19}$ However, in their retrospective study D'Angelo and colleagues $^{20}$ reported that adjuvant therapy with gefitinib or erlotinib was significantly associated with a lower risk of recurrence or death, disease-free survival, and a trend 
toward improved OS. In a randomized phase II study, adjuvant therapy with gefitinib following pemetrexed and carboplatin showed significantly improved disease-free survival in patients with stage IIIA NSCLC harboring EGFR mutations compared with pemetrexed and carboplatin treatment alone. ${ }^{21}$ However, in disagreement with our results, positive EGFR mutation status was recently identified as a favorable independent prognostic factor for survival periods following recurrence. ${ }^{22}$ Therefore, stratification by EGFR mutation status might be necessary to avoid an uneven distribution of $E G F R$ mutant populations that could potentially confound survival data in future clinical trials related to adjuvant therapy.

Our study must be interpreted in the context of several limitations. First, although the number of the EGFR mutant cohort was the largest evaluated to date, this study represents a retrospective comparative study at a single institution, and thus patient selection bias is inevitable. Second, the intrinsic prognostic role of EGFR mutation (ie, EGFR mutation as a prognostic factor) might not have been accurately evaluated, given that we could not completely exclude the efficacy of EGFR-TKI treatment (ie, EGFR mutation as a predictive factor); that is, our study did not include any patients who received EGFR-TKI treatment in the induction or adjuvant setting. Nevertheless, 41 of 939 patients $(4 \%)$ received EGFR-TKI treatment for postoperative recurrences. Third, although EGFR-TKI treatment for patients with recurrent $E G F R$-mutated tumors was not associated with a statistically significant increase in OS, the number of patients who received EGFR-TKI treatment for postoperative recurrence might be too small to allow for a definitive conclusion. Larger-scale studies in this population are warranted.

In conclusion, our analyses indicate that positive EGFR mutation status is a favorable prognostic factor in patients with surgically resected lung adenocarcinomas. Our results also show that EGFR mutation subtype (L858R mutation or exon19 deletions) has no prognostic impact, although it might be a significant predictor of EGFR-TKI efficacy (Video 1).

\section{Conflict of Interest Statement}

Authors have nothing to disclose with regard to commercial support.

\section{References}

1. Mitsudomi T, Kosaka T, Yatabe Y. Biological and clinical implications of EGFR mutations in lung cancer. Int J Clin Oncol. 2006;11:190-8.

2. Herbst RS, Fukuoka M, Baselga J. Gefitinib: a novel targeted approach to treating cancer. Nat Rev Cancer. 2004;4:956-65.

3. Lynch TJ, Bell DW, Sordella R, Gurubhagavatula S, Okimoto RA, Brannigan BW, et al. Activating mutations in the epidermal growth factor receptor underlying responsiveness of non-small-cell lung cancer to gefitinib. N Engl J Med. 2004;350:2129-39.

4. Paez JG, Jänne PA, Lee JC, Tracy S, Greulich H, Gabriel S, et al. EGFR mutations in lung cancer: correlation with clinical response to gefitinib therapy. Science. 2004;304:1497-500.
5. Zhang Y, Sheng J, Kang S, Fang W, Yan Y, Hu Z, et al. Patients with exon 19 deletion were associated with longer progression-free survival compared to those with L858R mutation after first-line EGFR-TKIs for advanced non-small cell lung cancer: a meta-analysis. PLoS One. 2014;9:e107161.

6. Zhang Z, Wang T, Zhang J, Cai X, Pan C, Long Y, et al. Prognostic value of epidermal growth factor receptor mutations in resected non-small cell lung cancer: a systematic review with meta-analysis. PLoS One. 2014;9:e106053.

7. Lee YJ, Park IK, Park MS, Choi HJ, Cho BC, Chung KY, et al. Activating mutations within the EGFR kinase domain: a molecular predictor of disease-free survival in resected pulmonary adenocarcinoma. J Cancer Res Clin Oncol. 2009; 135:1647-54.

8. Liu WS, Zhao LJ, Pang QS, Yuan ZY, Li B, Wang P. Prognostic value of epidermal growth factor receptor mutations in resected lung adenocarcinomas. Med Oncol. 2014;31:771.

9. Okamoto T, Kitahara H, Shimamatsu S, Katsura M, Takada K, Fujishita T, et al. Prognostic impact of EGFR driver mutations on postoperative disease recurrence in lung adenocarcinoma. Anticancer Res. 2016;36:3057-63.

10. Goldstraw P, Crowley J, Chansky K, Giroux DJ, Groome PA, Rami-Porta R, et al. The IASLC Lung Cancer Staging Project: proposals for the revision of the TNM stage groupings in the forthcoming (seventh) edition of the TNM Classification of Malignant Tumours. J Thorac Oncol. 2007;2:706-14.

11. Nagai $Y$, Miyazawa $H$, Huqun, Tanaka $T$, Udagawa $K$, Kato $M$, et al. Genetic heterogeneity of the epidermal growth factor receptor in non-small cell lung cancer cell lines revealed by a rapid and sensitive detection system, the peptide nucleic acid-locked nucleic acid PCR clamp. Cancer Res. 2005;65: 7276-82.

12. Kobayashi S, Boggon TJ, Dayaram T, Jänne PA, Kocher O, Meyerson M, et al. EGFR mutation and resistance of non-small-cell lung cancer to gefitinib. N Engl J Med. 2005;352:786-92.

13. Steuer CE, Khuri FR, Ramalingam SS. The next generation of epidermal growth factor receptor tyrosine kinase inhibitors in the treatment of lung cancer. Cancer. 2015;121:E1-6.

14. Govindan R, Ding L, Griffith M, Subramanian J, Dees ND, Kanchi KL, et al. Genomic landscape of non-small cell lung cancer in smokers and never-smokers. Cell. 2012;150:1121-34.

15. Goldstraw P, Chansky K, Crowley J, Rami-Porta R, Asamura H, Eberhardt WE, et al. The IASLC Lung Cancer Staging Project: proposals for revision of the TNM stage groupings in the forthcoming (eighth) edition of the TNM Classification for Lung Cancer. J Thorac Oncol. 2016;11:39-51.

16. Hirsch FR, Varella-Garcia M, Bunn PA Jr, Franklin WA, Dziadziuszko R, Thatcher N, et al. Molecular predictors of outcome with gefitinib in a phase III placebo-controlled study in advanced non-small-cell lung cancer. J Clin Oncol. 2006;24:5034-42.

17. Mok TS, Wu YL, Thongprasert S, Yang CH, Chu DT, Saijo N, et al. Gefitinib or carboplatin-paclitaxel in pulmonary adenocarcinoma. N Engl J Med. 2009;361: 947-57.

18. Goss GD, O’Callaghan C, Lorimer I, Tsao MS, Masters GA, Jett J, et al. Gefitinib versus placebo in completely resected non-small-cell lung cancer: results of the NCIC CTG BR19 study. J Clin Oncol. 2013;31:3320-6.

19. Kelly K, Altorki NK, Eberhardt WE, O’Brien ME, Spigel DR, Crinò L, et al. Adjuvant erlotinib versus placebo in patients with stage IB-IIIA non-small-cell lung cancer (RADIANT): a randomized, double-blind, phase III trial. J Clin Oncol. 2015;33:4007-14.

20. D'Angelo SP, Janjigian YY, Ahye N, Riely GJ, Chaft JE, Sima CS, et al. Distinct clinical course of EGFR-mutant resected lung cancers: results of testing of 1118 surgical specimens and effects of adjuvant gefitinib and erlotinib. J Thorac Oncol. 2012;7:1815-22.

21. Li N, Ou W, Ye X, Sun HB, Zhang L, Fang Q, et al. Pemetrexed-carboplatin adjuvant chemotherapy with or without gefitinib in resected stage IIIA-N2 non-small cell lung cancer harbouring EGFR mutations: a randomized, phase II study. Ann Surg Oncol. 2014;21:2091-6.

22. Kudo Y, Shimada Y, Saji H, Kato Y, Yoshida K, Matsubayashi J, et al. Prognostic factors for survival after recurrence in patients with completely resected lung adenocarcinoma: important roles of epidermal growth factor receptor mutation status and the current staging system. Clin Lung Cancer. 2015;16:e213-21.

Key Words: lung adenocarcinoma, epidermal growth facter receptor, gene mutation, prognosis 
OS

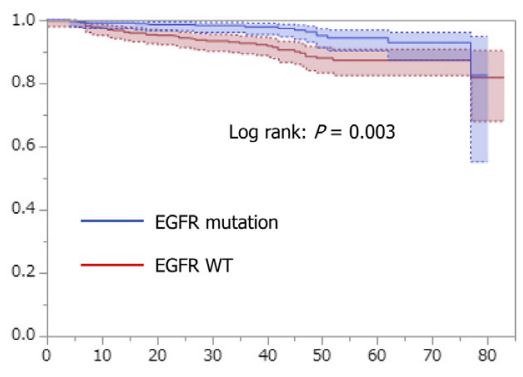

A EGFR mutation
35
350
391

$\begin{array}{ll}335 & 219 \\ 360 & 220\end{array}$

83
96

B EGFR mutation

B EGFR mutation

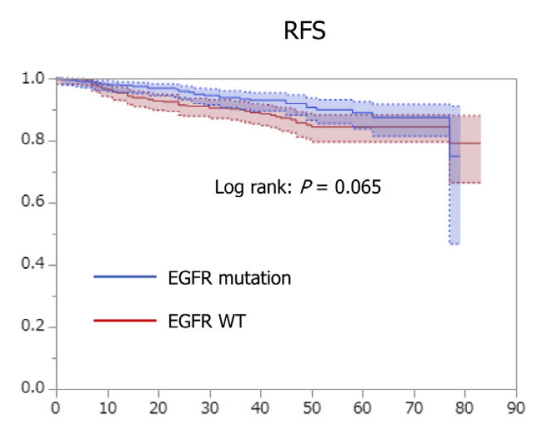

FIGURE E1. Overall survival (A) and recurrence-free survival (B) in patients with pathological stage I lung adenocarcinomas according to EGFR mutation status. OS, Overall survival; RFS, recurrence-free survival; EGFR, epidermal growth factor receptor; WT, wild type.

OS

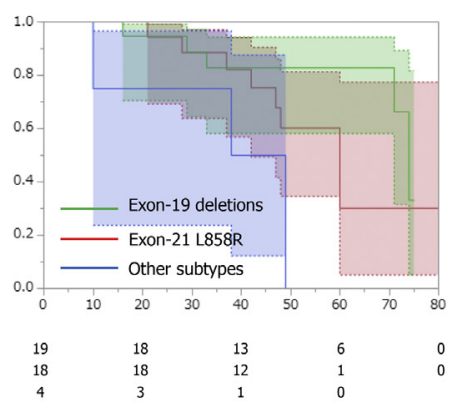

Survival periods following recurrence

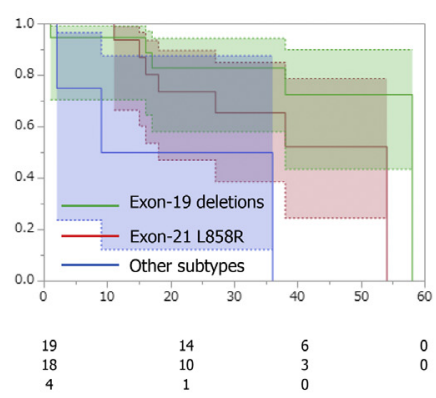

FIGURE E2. Overall survival (A) and survival periods following recurrence (B) in patients who received epidermal growth factor receptor (EGFR) tyrosine-kinase inhibitor TKI treatment according to EGFR mutation subtype (exon 19 deletions, exon 21 L858R mutation, or other mutations). OS, Overall survival. 